Ann. Biol. anim. Bioch. Biophys., I97I, 11 (I), I67-I74.

\title{
MAINTIEN ET RESTAURATION DE LA SPERMATOGENESE PAR UN EXTRAIT ACÉTONIQUE HYPOPHYSAIRE DE CARPE CHEZ LE CYPRIN (CARASSIUS AURATUS) TRAITÉ AU MÉTHALLIBURE
}

\author{
R. BILLARD, B. BRETON et Anne-Marie ESCAFFRE \\ Station centrale de Physiologie animale, \\ Centre national de Recherches zootechniques, I. N.R. A., \\ 78 - Jouy-en-Josas
}

\begin{abstract}
RÉSUMÉ
Le mëthallibure administré en bains à la dose de 2 p.p.m. entraîne chez le poisson rouge (Carassius auratus) une inhibition de la spermatogenèse qui intéresse toutes les cellules germinales à l'exception des spermatogonies A. Lorsque les mâles soumis à ce traitement reçoivent en même temps des injections de poudre hypophysaire brute de Carpe, la spermatogenèse est maintenue et même fortement stimulée.

Après la suppression du traitement au méthallibure, la spermatogenèse se rétablit complètement en 20 jours, mais la restauration est plus importante si les animaux reçoivent des injections de poudre hypophysaire brute de Carpe.
\end{abstract}

\section{INTRODUCTION}

Le méthallibure (ICI 33 828) est fréquemment utilisé pour bloquer le fonctionnement gonadotrope hypophysaire chez les Mammifères (PAGET et al., I96r ; HEMSWORTH et al., I968; WALPOLE, I968 ; GARBERS et FIRST, I969).

Ce produit présente également la propriété d'inhiber la spermatogenèse des poissons ; chez Gasterosteus acculeatus et Carassius auratus (HOAR et al., I967), chez Cymatogaster aggregata (HOAR et al., I967; WEIBE, I968-I969), chəz Poecilia reticulata (MARTin et BROMAGE, I970 ; PANDEY et LEATHERLAND, I970; PANDEY, I970 ; BIILARD et al., I970). Chez Cymatogaster aggregata une étude ultrastructurale de 1'hypophyse (LEATHERLAND, I969) permet de penser que le méthallibure bloque la synthèse des hormones gonadotropes au niveau du système hypothalamo-hypophy- 
saire. Dans ces conditions la gamétogenèse ne devrait pas être altérée par l'administration simultanée d'hormones gonadotropes et de méthallibure. La seule tentative faite dans ce sens est due à WIEBE (I968, r969) mais 1'impossibilité de maintenir le poids testiculaire peut être due au facteur gonadotrope utilisé $\left(\mathrm{L}_{\mathrm{L}} \mathrm{H}\right.$ ovine). Dans le présent travail, une expérience analogue est conduite en utilisant une préparation acétonique brute d'hypophyse de Carpe.

\section{MATÉRIEL, ET MÉTHODES}

Les Carassius, d'un poids de 30 à $50 \mathrm{~g}$, proviennent de la souche élevée au laboratoire et sont placés par lots de 4 dans des aquarium de 201 . L'eau qui est renouvellée par moitié chaque semaine est maintenue au voisinage de la saturation en oxygène, grâce à la diffusion d'air comprimé. Les animaux sont placés sous photopériode de $12 \mathrm{~L}-\mathbf{I} 2 \mathrm{~N}$ et à la température constante de $\mathrm{I} 7^{\circ} \mathrm{C}$. L'expérience débute au mois d'octobre. Le méthallibure maintenu en suspension par le Triton $\times$ roo est administré en bains, tous les jours, à la dose de 2 p.p.m., selon le processus déjà décrit (BILlARD et al., I970).

Cinq groupes d'animaux reçoivent les traitements suivants (fig. I) :

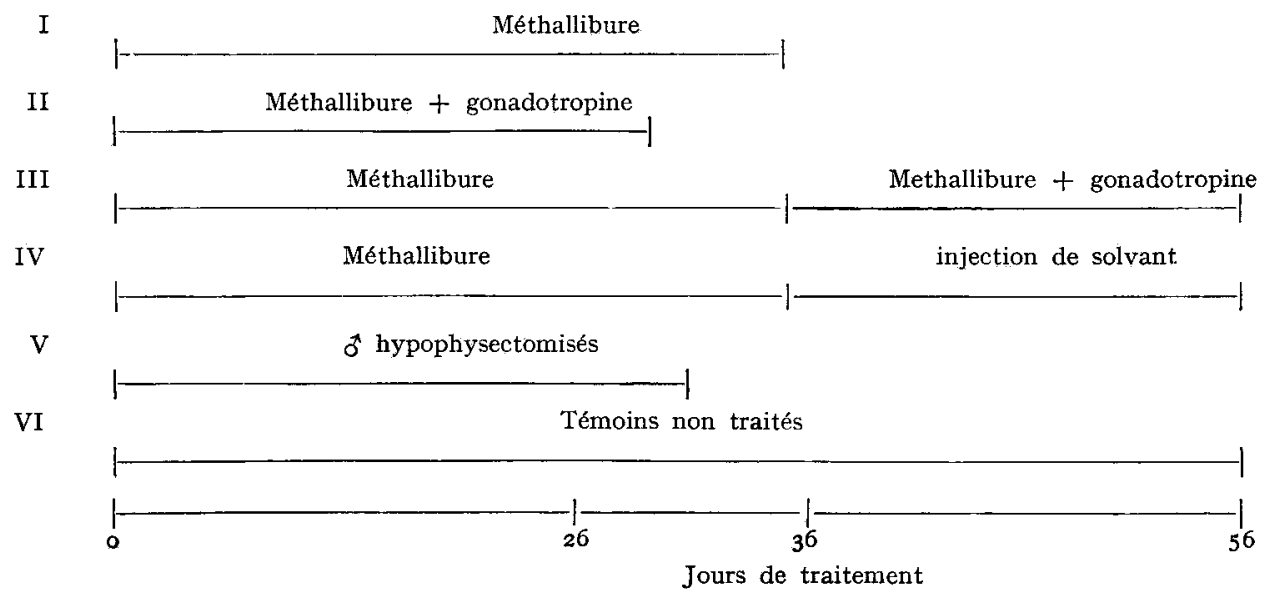

FIG. I. - Schéma de l'expérience

Groupe I: animaux témoins traités au méthallibure

Le méthallibure est administré seul et le traitement est prolongé jusqu'à la régression complète des spermatozoïdes dans les testicules, c'est-à-dire pendant 36 jours.

\section{Groupe II: maintien de la spermatogenèse}

Les animaux reçoivent d'une part le traitement au méthallibure, qui est administré en bains, et d'autre part des injections intrapéritonéales de poudre hypophysaire de Carpe (Io $\gamma / g$ poids vif et par injection) ou de HCG (Io UI/g poids vif et par injection). L'expérience conduite jusqu'à ce que le poids des gonades atteigne $4 \mathrm{p}$. Ioo du poids du corps (valeur observée pendant la période de reproduction au printemps) dure 26 jours et les animaux reçoivent au total so injections ( 3 injections par semaine).

\section{Groupe III: régénération de la spermatogenèse}

Après 36 jours de traitement, l'administration de méthallibure est maintenue, mais les animaux reçoivent en même temps le traitement gonadotrope dans les mêmes conditions que précédemment pendant 20 jours, soit 8 injections. 
Groupe IV : végénération de la spermatogenèse après la fin du traitement au méthallibure

L'administration du méthallibure est supprimée après 36 jours de traitement ; le milieu est complètement renouvelé et pendant les 20 jours suivants les poissons reçoivent seulement des injections de solvant.

Dans les groupes III et IV l'administration des gonadotropines est maintenue jusqu'à l'émission des spermatozoïdes (après massage abdominal) qui intervient au $20^{\mathbf{e}}$ jour.

\section{Groupe V: animaux hypophysectomisés}

Groupe VI: animaux témoins ne recevant ni drogues ni hormones

Les groupes $\mathrm{I}$ et $\mathrm{V}$ comportent 8 animaux et les autres 4. L'administration des gonadotropines est réalisée selon le processus déjà décrit (BILlaRD et al., r970).

L'hypophysectomie est pratiquée selon la technique de CHAVIN (I956) modifiée par LaHLou et SAWYER (r969). L'effet du traitement est estimé par le taux de mortalité, la variation du poids corporel au cours de l'expérience, le rapport gonadosomatique (RGS), le volume occupé par les différentes catégories de cellules germinales dans le testicule dont l'évaluation est faite selon le processus déjà décrit (BILlARD et al. I97I b), et la spermiation (émission de spermatozoïdes après massage abdominal). Les techniques histologiques comportent la fixation des testicules dans le fixateur Bouin-Hollande et l'exécution de coupes sériées de $5 \mu$.

\section{RÉSULTATS}

Les résultats sont résumés dans la figure 2 .

\section{Action comparée du méthallibure et de l'hypophysectomie sur la spermatogenèse (groupes I et V)}

Le méthallibure administré en bains entraîne une diminution du RGS et de toutes les catégories cellulaires dans le testicule, à l'exception des spermatogonies A dont le nombre reste stationnaire au cours de l'expérience : les spermatogonies $\mathrm{B}$, les spermatocytes et les spermatides sont les catégories cellulaires les plus sensibles. Le volume occupé par les lumières des lobules diminue également. La réduction de la quantité de spermatozoïdes n'est sensible qu'au-delà du $26^{\mathrm{e}}$ jour du traitement. Après hypophysectomie chirurgicale, les résultats obtenus sont comparables mais la décroissance de certaines catégories cellulaires (spermatides et spermatozoïdes) est légèrement plus rapide.

Action des hormones gonadotropes exogènes administrées en même temps que le méthallibure

(groupe II et III)

La poudre hypophysaire de Carpe administrée simultanément avec le méthallibure maintient et stimule même la spermatogenèse par rapport à la situation de départ. Dans l'expérience de maintien, l'injection de poudre hypophysaire entraîne une diminution du nombre de spermatides et de spermatozoïdes vraisemblablement due à l'initiation des processus de spermiation qui se produisent dès le $12^{\mathbf{e}}$ jour et qui affectent les générations de spermatides et de spermatozoïdes présents dans le testicule au début de l'expérience. 
FiG. 2. - Action comparêe de l'hypophysectomie et du méthallibure administré seul ou en combinaison avec de la poudre hypophysaire totale de Carpe et d'HCG sur la spermatogenèse du poisson rouge

Dans le cas du RGS le poids des gonades est exprimé en pourcentage du poids du corps.

La quantité de cellules germinales est exprimée en volume occupé par les différents types cellulaires dans la totalité du testicule. Ces résultats sont ramenés au gramme de poids corporel et concernent les cellules de Sertoli (Sertoli), les spermatogonies A (GA) et B (GB), les spermatocytes I (Scytes), les spermatides (Sptides) les spermatozoides (Spz) et la lumière des lobules non occupée par des spermatozoides. - Animaux témoins au départ de l'expérience

Animaux traités au methallibure

méthallibure administré seul (Groupe I)

méthallibure administré conjointement avec :

$\square$ de la poudre hypophysaire totale de Carpe expérience de maintien (groupe II) expérience de régénération (groupe III) $\triangle$ ou de HCG (Intervet)

Animaux hypophysectomisés (groupe V)

- Régénération de la spermatogenèse après cessation du traitement au méthallibure (groupe IV)

* Spermiation (émission des spermatozoïdes après massage abdominal)

Les chiffres indiqués sur le graphique du haut correspondent au nombre d'animaux exploités. 

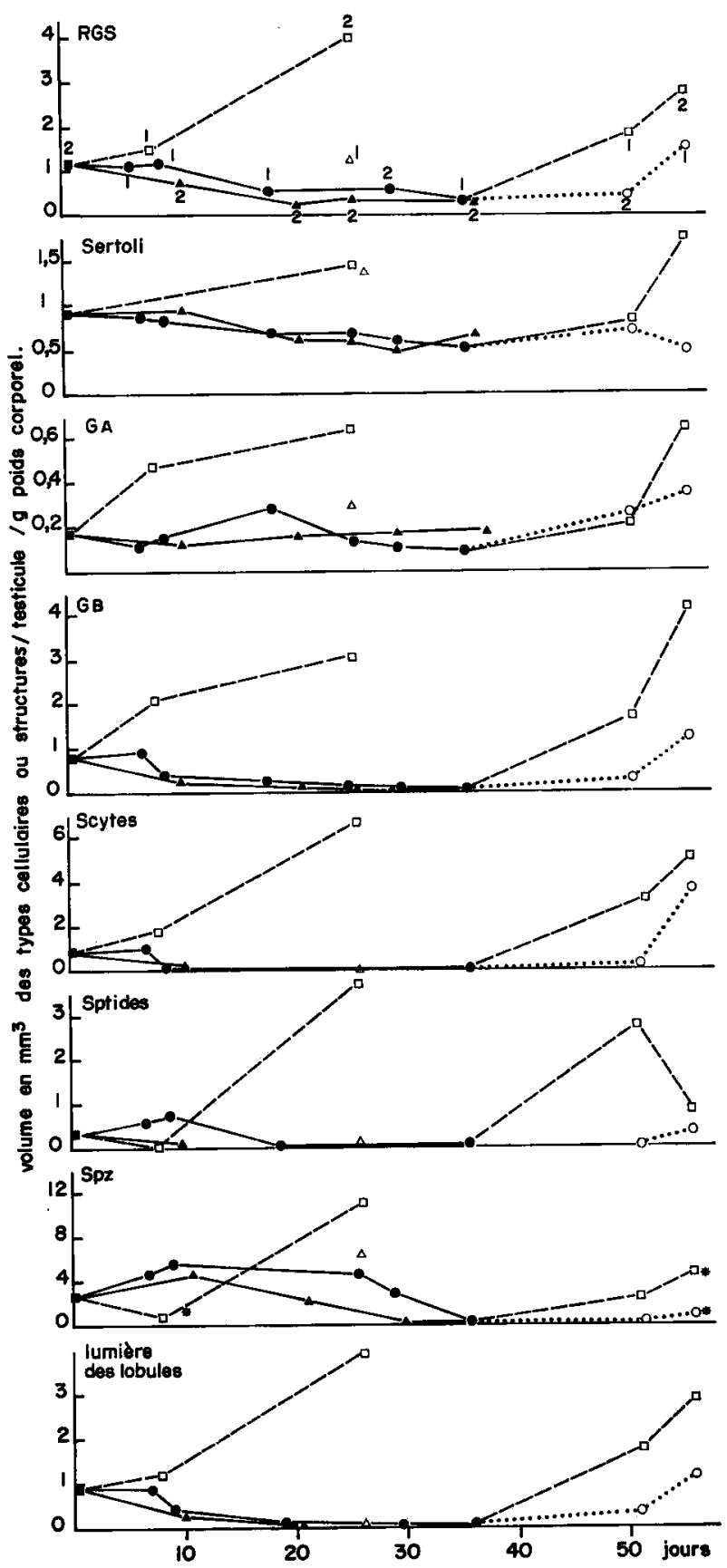
Dans l'expérience de régénération les résultats sont comparables ; la diminution du nombre de spermatides après I 5 jours de traitement peut également être mise en relation avec les formations des spermatozoïdes et la spermiation. Cette dernière intervient après 20 jours de traitement gonadotrope.

\section{Régénération de la spermatogenèse}

après cessation du traitement au méthallibure (groupe IV)

Après un traitement de 36 jours au méthallibure, l'activité spermatogénétique qui est fortement inhibée se rétablit normalement et dans les 20 jours qui suivent, la spermiation a lieu. Cependant 1'initiation de la spermatogenèse est plus lente et l'importance de la régénération reste plus faible qu'après les supplémentations hormonales faites pendant la même période dans le groupe III.

\section{Action du méthallibure sur la croissance et la survie des animaux}

La mortalité due au méthallibure est pratiquement nulle. Dans la plupart des cas, les animaux subissent, au cours de l'expérience, une légère perte de poids qui n'excède pas 3 à $4 \mathrm{p}$. Ioo du poids du corps, mais une perte de poids du même ordre se retrouve également chez les animaux témoins (groupe VI) de sorte que l'amaigrissement n'est pas dû à une action directe du méthallibure mais plutôt au confinement des animaux.

\section{Action de $H C G$}

HCG, administré pendant 26 jours en même temps que le méthallibure, maintient le poids testiculaire mais l'analyse histologique montre que seules les spermatogonies A, les cellules de Sertoli (fig. 2) et le conjonctif extralobulaire sont maintenus à leur niveau normal ; il n'y a plus de spermatocytes et de spermatides.

L'activité spermatogénétique des animaux témoins (groupe VI) reste la même pendant toute la durée de l'expérience.

\section{DISCUSSION E'T CONCLUSION}

L'inhibition de la spermatogenèse de Carassius auratus par le méthallibure confirme les résultats de HOAR et al. (I967) et la comparaison avec les effets dus à l'hypophysectomie montre que les mêmes catégories cellulaires sont affectées dans les deux cas. Dans cette espèce les effets du méthallibure sont sensiblement aussi rapides qu'après hypophysectomie chirurgicale alors que chez Poecilia reticulata l'action du méthallibure est plus lente (BILLARD et al., I970).

Le méthallibure n'empêche pas l'action des hormones gonadotropes exogènes, ce qui prouve que l'action de ce produit se situe au niveau du complexe hypothalamohypophysaire comme chez les Mammifères (BRown, I963; HEMsworTH et al.,Ig68).

HCG qui est considéré comme gonadotrope chez certains poissons (FONTAINE, I936; AsHAN et HOAR, I963) est incapable de maintenir chez le poisson rouge cer- 
taines catégories de cellules germinales telles que spermatogonies B, spermatocytes et spermatides.

Chez Carassius auratus, le méthallibure peut donc être utilisé pour bloquer le fonctionnement hypophysaire dans les expériences de supplémentation hormonales gonadotropes.

Reçu pour publication en octobre 1970.

\section{REMERCIEMENTS}

Le méthallibure nous a aimablement été fourni par Imperial Chemical Industries, Macclesfield, England.

La poudre hypophysaire de Carpe utilisée dans l'expérience provient d'un prélèvement assuré

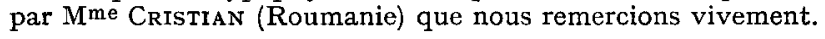

\section{SUMMARY}

\section{EFFECT OF A CARP PITUITARY ACETONIC EXTRACT ON SPERMATOGENESIS IN THE " METHALLIBURE " TREATED GOLDFISH CARASSIUS AURATUS}

Administration of methallibure in the water at 2 p.p.m. rates inhibited the spermatogenesis of the goldfish Carassius auratus at every stage of the germinal cells except A-spermatogonia. The same results were obtained by means of surgical hypophysectomy.

Spermatogenesis was maintained or increased, when the methallibure treated animals are injected gonadotrophic hormone in the form of Carp total pituitary extract. Spermatogenesis was thoroughly resumed with male fish whose gonads were regressed by 36 days of methallibure treatment. Spermiation occured after 20 days.

The spermatogenesis of non injected fish was normally resumed and spermiation occured within 20 days after cessation of the methallibure treatment.

\section{RÉFÉRENCES BIBLIOGRAPHIQUES}

Ahsan S. N., Hoar W. J., r963. Some effects of gonadotropic hormones on the threespine stickleback Gasterosteus aculeatus. Can. J. Zool., 41, r045-1053.

Biliard R., Burzawa-Gérard E., Breton B., I97o. Régénération de la spermatogenèse du cyprin hypophysectomisé Carassius auratus par un facteur gonadotrope hautement purifié de carpe. $C$. $R$. Acad. Sci., D, 271, I896-1899.

Billard R., Breton B., Jalabert B., I970. Inhibition de la spermatogenèse du Guppy (poisson cyprinodontidae) par le méthallibure. Ann. Biol. anim. Bioch. Biophys., 10, 5II-5 5 .

Billard R. Breton Bet Solari A, r97r. Les méthodes d'analyse de la spermatogenèse des poissons téléostéens. Ann. Biol. anim. Bioch. Biophys., (à paraître).

BRown P. S., r963. Observations on a dithio-carbamoylhydrazine as an inhibitor of pituitary gonadotropic activity. J. Endocr., 26, 425-436.

Chavin W., r956. Pituitary adrenal control of melanization in xantic Goldfish Carassius auratus L. J. Exptl. Zool., I33-146.

Fontaine M., I936. Sur la maturation complète des organes génitaux de l'anguille mâle et l'émission spontanée de ses produits sexuels. C. R. Acad. Sci., 202, 13I2-1314.

Garbers D. L., First N. L., I969. The effects of injected cestradiol I7 B progesterone and dietary ICI 33828 on ovarian and pituitary functions in the sow and gilt. J. Reprod. Fert., 20, 45I-464-

Hoar W. S., Wiebe J., WaI E. H., I967. Inhibition of the pituitary gonadotrophin activity of fishes by a dithiocarbamoylhydrazine derivaritive (ICI 33 828). Gen. Comp. Endocr., 8, roI-Iog. 
Hemsworth B. N., Jackson H., Walpole A. L., I968. Effect of the pituitary inhibitor ICI 33828 (methallibure) on the reproductive system of male rats. J. Endocr., 40, 275-284.

LAHLOU B., SAWYER W. H., 1969. Electrolyte balance in hypophysectomized goldfish Carassine auratus. Gen. Comp. Endocr., 12, 370-377.

Leatherland J. F., I969. Studies on the structure and ultrastructure of the intact and " methallibure "treated meso-adenohypophysis of the Viviparous Teleost Cymatogaster aggregata GibBons. $Z$. Zellforsch. 98, $122-134$.

Martin P., Bromage N. R., I970. The effect of methallibure on spermatogenesis in Poecilia reticulata. J. Fish Biol., 2, 47-5r.

Paget G. E., Walpore A. L, Richardson D. N., Ig6r. Non steroidal inhibitors of pituitary gonadotrophic function. Nature, 192, II9r.

Pandey S., 197o. Effects of methallibure on the testes and secondary sex characters of the adult and juvenile Guppy Poecilia. reticulata PeTERs. Biol. Reprod.; 2, 239-244.

Pandey S., Leatherland J. F., r97o. Comparison of the effects of methallibure and thiourea on the testis thyroid and adenohypophysis of the adult and juvenile guppy Poecilia reticulata PETERs. Can. $J$. Zool., 48, 445-450.

WALPOLE A. L., I968. Non steroidal drugs in relation to ovulation and implantation. J. Reprod. Fert. Suppl., 4, 3-14.

WIEBE J. P., I968. Inhibition of pituitary gonadotrophic activity in the viviparous seaperch Cymatogaster aggregata GIBBoNs by a dithiocarbamoylhydrazine derivative ICI 33828 . Can. J. Zool., 46, $75 \mathrm{I}-758$.

WIEBE J. P., I969. Endocrine controle of spermatogenesis and oogenesis in the viviparous seaperch Cymatogaster aggregata GiBBoNs. Gen. Comp. Endocr., 12, 267-275. 